\title{
Maps to the projective plane
}

\author{
JERZY DYDAK \\ MiCHAEL LEVIN
}

\begin{abstract}
We prove the projective plane $\mathbb{R} P^{2}$ is an absolute extensor of a finite-dimensional metrizable space $X$ if and only if the cohomological dimension mod 2 of $X$ does not exceed 1. This solves one of the remaining difficult problems (posed by A N Dranishnikov) in Extension Theory. One of the main tools is the computation of the fundamental group of the function space $\operatorname{Map}\left(\mathbb{R} P^{n}, \mathbb{R} P^{n+1}\right.$ ) (based at the inclusion) as being isomorphic to either $\mathbb{Z}_{4}$ or $\mathbb{Z}_{2} \oplus \mathbb{Z}_{2}$ for $n \geq 1$. Double surgery and the above fact yield the proof.
\end{abstract}

$54 \mathrm{~F} 45 ; 54 \mathrm{C} 65,55 \mathrm{M} 10$

\section{Introduction}

The basic relation studied by Extension Theory is that of a CW-complex $K$ being an absolute extensor of a metrizable space $X$. This means that every map $f: A \rightarrow K, A$ closed in $X$, extends continuously over $X$. There are three existing notations for this:

(1) $K \in \operatorname{AE}(X)$.

(2) $X \tau K$.

(3) $\operatorname{edim} X \leq K$.

In addition, we will use the notation $X \tau(L \rightarrow K)$, where $L$ is a subcomplex of $K$. This means that every map $f: A \rightarrow L, A$ closed in $X$, extends continuously over $X$ with values in $K$. See Cencelj et al [4] for more information about this relation.

In the case of basic CW-complexes, the relation $X \tau K$ is equivalent to classical concepts in dimension theory (see Dranishnikov [7] for more details):

(a) $X \tau S^{n}$, for $S^{n}$ the $n$-sphere, is equivalent to covering $\operatorname{dimension} \operatorname{dim}(X)$ being at most $n$.

(b) $X \tau K(G, n)$, for $K(G, n)$ an Eilenberg-Mac Lane complex, is equivalent to the cohomological dimension $\operatorname{dim}_{G}(X)$ being at most $n$.

One of the leading themes in Extension Theory is the effort to relate $X \tau K$ to a set of conditions $X \tau K\left(G_{n}, n\right)$, where $G_{n}$ depends on $K$. In that vein Dranishnikov [6] 
proved the following important theorems connecting extensional and cohomological dimensions of compacta (ie, metrizable compact spaces).

Theorem 1.1 Let $K$ be a $C W$-complex and $X$ be a compactum such that edim $X \leq K$. Then $\operatorname{dim}_{H_{n}(K)}(X) \leq n$ for every $n>0$.

Theorem 1.2 Let $K$ be a simply connected $C W$-complex and let $X$ be compactum that is finite-dimensional. If $\operatorname{dim}_{H_{n}(K)}(X) \leq n$ for every $n>0$, then $\operatorname{edim} X \leq K$.

Both Theorem 1.1 and Theorem 1.2 were subsequently generalized for metrizable spaces $X$ in Dydak [9]. The requirement in Theorem 1.2 that $X$ is finite-dimensional cannot be omitted. To show this, take the famous infinite-dimensional compactum $X$ of Dranishnikov with $\operatorname{dim}_{\mathbf{Z}}(X)=3$ as in [5]. Then the conclusion of Theorem 1.2 does not hold for $K=S^{3}$. In the absence of the finite-dimensionality of $X$ the following example from Levin [11] may serve as a source of many counterexamples: there is a compactum $X$ satisfying the following conditions:

(a) $\operatorname{edim} X>K$ for every finite CW-complex $K$ with $\tilde{H}_{*}(K) \neq 0$.

(b) $\operatorname{dim}_{G} X \leq 2$ for every abelian group $G$.

(c) $\operatorname{dim}_{G} X \leq 1$ for every finite abelian group $G$.

Here $\operatorname{edim} X>K$ means that $\operatorname{edim} X \leq K$ is false.

With no restriction on $K$, Theorem 1.2 does not hold. Indeed, the conclusion of Theorem 1.2 is not satisfied if $K$ is a noncontractible acyclic CW-complex and $X$ is the 2dimensional disk. Cencelj and Dranishnikov [2] generalized Theorem 1.2 for nilpotent CW-complexes $K$ and $X$ being a compactum (see Cencelj and Dranishnikov [1] for the case of $K$ with finitely generated fundamental group ). Their work was generalized by Cencelj, Dydak, Mitra and Vavpetič [3] to metrizable $X$.

The real projective plane $\mathbb{R} P^{2}$ is the simplest CW-complex not covered by Cencelj and Dranishnikov's result. Thus we arrive at the following well-known open problem in Extension Theory.

Problem 1.3 Let $X$ be a finite-dimensional compactum. Does $\operatorname{dim}_{\mathbf{Z}_{2}}(X) \leq 1$ imply $\operatorname{edim} X \leq \mathbb{R} P^{2}$ ?

A partial answer to Problem 1.3 was given by the authors in [10]:

Theorem 1.4 Let $X$ be a compactum of dimension at most three. If $\operatorname{dim}_{\mathbf{Z}_{2}}(X) \leq 1$, then $\operatorname{edim} X \leq \mathbb{R} P^{2}$. 
This paper is devoted to solving Problem 1.3 completely and in the affirmative - see Corollary 4.8. In view of this it is of interest to address the following question:

Problem 1.5 Let $X$ be a compactum of finite dimension. Does $\operatorname{dim}_{\mathbf{Z}_{p}}(X) \leq 1$ imply $\operatorname{edim}(X) \leq M\left(\mathbf{Z}_{p}, 1\right)$ for any Moore complex $M\left(\mathbf{Z}_{p}, 1\right)$ with $\pi_{1}\left(M\left(\mathbf{Z}_{p}, 1\right)\right)=\mathbf{Z}_{p}$ ?

Our models of projective spaces are as follows:

- One is the quotient space of the unit sphere $S^{n}$ in a normed space $V^{n+1}$ isometric to the Euclidean $(n+1)$-dimensional space $\mathbb{R}^{n+1}$. As usual, the antipodal points on the unit sphere are identified. $p_{n}: S^{n} \rightarrow \mathbb{R} P^{n}$ is the quotient map.

- The second is the quotient space of the unit ball $B^{n}$ in a normed space $V^{n}$ isometric to the Euclidean $n$-dimensional space $\mathbb{R}^{n}$. As usual, the antipodal points on the unit sphere are identified.

- The third is the quotient space of the upper hemisphere $B^{n}$ of $S^{n}$ onto $\mathbb{R} P^{n}$. The antipodal points on the unit sphere $S^{n-1}$ are identified.

In the second and the third models we will use the same symbol $p_{n}$ to denote the quotient map $p_{n}: B^{n} \rightarrow \mathbb{R} P^{n}$.

It will be clear from the context which model we have in mind in a particular situation.

By writing $\mathbb{R} P^{m} \subset \mathbb{R} P^{n}$ or saying that $\mathbb{R} P^{m}$ is in $\mathbb{R} P^{n}$ we mean that the pair $\left(\mathbb{R} P^{n}, \mathbb{R} P^{m}\right.$ ) is diffeomorphic to the one obtained from the first model of $\mathbb{R} P^{n}$ with $\mathbb{R} P^{m}=p_{n}\left(S^{n} \cap V^{m+1}\right)$ and $V^{m+1}$ an $(m+1)$-dimensional linear subspace of $V^{n+1}$. Similarly for a collection of projective spaces $\mathbb{R} P^{m_{1}}, \ldots, \mathbb{R} P^{m_{k}}$ in $\mathbb{R} P^{n}$ we always assume that up to a diffeomorphism of $\mathbb{R} P^{n}$ the collection can be represented based on the first model of $\mathbb{R} P^{n}$ so that $\mathbb{R} P^{m_{i}}=p_{n}\left(S^{n} \cap V^{m_{i}+1}\right)$ with $V^{m_{i}+1}$ a linear subspace of $V^{n+1}$. Note that an increasing sequence $\mathbb{R} P^{m_{1}} \subset \cdots \subset \mathbb{R} P^{m_{k}} \subset \mathbb{R} P^{n}$ is defined uniquely up to a diffeomorphism of $\mathbb{R} P^{n}$ (induced by an orthogonal transformation of $\left.V^{n+1}\right)$.

It is easy to see that in the second model of $\mathbb{R} P^{n}$ we can represent $\mathbb{R} P^{n-1}$ in $\mathbb{R} P^{n}$ as $\mathbb{R} P^{n-1}=p_{n}\left(\partial B^{n}\right)$ and we can also represent $\mathbb{R} P^{m}$ in $\mathbb{R} P^{n}$ as $\mathbb{R} P^{m}=p_{n}\left(B^{n} \cap V^{m}\right)$ with $V^{m}$ being an $m$-dimensional linear subset of $V^{n}$. Specifically for the last interpretation of $\mathbb{R} P^{m} \subset \mathbb{R} P^{n}$ we define the projective space $\mathbb{R} P_{\perp}^{n-m} \subset \mathbb{R} P^{n}$ orthogonal to $\mathbb{R} P^{m}$ as $\mathbb{R} P_{\perp}^{n-m}=p_{n}\left(B^{n} \cap V_{\perp}^{n-m}\right)$ with the linear subspace $V_{\perp}^{n-m}$ the orthogonal complement of $V^{m}$.

We use the convention $\mathbb{R} P^{m}=\varnothing$ if $m<0$. 
Acknowledgements The authors are grateful for the referee's attention to detail. As a result, the paper has been significantly improved.

This research was supported by Grant No. 2004047 from the United States-Israel Binational Science Foundation (BSF), Jerusalem, Israel. The first-named author was partially supported by the Center for Advanced Studies in Mathematics at Ben Gurion University of the Negev (Beer-Sheva, Israel).

\section{The fundamental group of function spaces}

Consider the inclusion $i: \mathbb{R} P^{n} \rightarrow \mathbb{R} P^{n+1}$. We are interested in the fundamental group of the space $\operatorname{Map}\left(\mathbb{R} P^{n}, \mathbb{R} P^{n+1}\right)$ of maps from $\mathbb{R} P^{n}$ to $\mathbb{R} P^{n+1}$ with $i$ as a base point. For $n \geq 1$ there is a homomorphism

$$
\lambda: \pi_{1}\left(\operatorname{Map}\left(\mathbb{R} P^{n}, \mathbb{R} P^{n+1}\right), i\right) \rightarrow \pi_{1}\left(\mathbb{R} P^{n+1}\right) \approx \mathbf{Z}_{2}
$$

induced by the evaluation map $e: \operatorname{Map}\left(\mathbb{R} P^{n}, \mathbb{R} P^{n+1}\right) \rightarrow \mathbb{R} P^{n+1}$ evaluated at $\mathbb{R} P^{0}$. The goal of this section is to show $\lambda$ is an epimorphism whose kernel is $\mathbf{Z}_{2}$.

Any loop $f: S^{1} \rightarrow \operatorname{Map}\left(\mathbb{R} P^{n}, \mathbb{R} P^{n+1}\right)$ that is based at $i$ can be converted to a map $\alpha: \mathbb{R} P^{n} \times S^{1} \rightarrow \mathbb{R} P^{n+1}$ such that $\alpha \mid\left(\mathbb{R} P^{n} \times 1\right)$ is the inclusion; in turn such $\alpha$ determines the loop $f$. Then $[f]$ is in the kernel of $\lambda$ if and only if $\alpha \mid\left(\mathbb{R} P^{0} \times S^{1}\right)$ is null-homotopic. Our initial objective is to show that $\alpha$ can be chosen to be in normal form (see Corollary 2.3). First, we will need a few preliminary results.

Lemma 2.1 Suppose $K$ is a connected $C W$-complex, $L$ is a connected subcomplex of $K$, two maps $f, g: K \rightarrow \mathbb{R} P^{n+1}$ agree on $L$ for some $n \geq 0$, and $\pi_{1}(f)=\pi_{1}(g)$. $f$ and $g$ are homotopic rel. $L$ if $\operatorname{dim}(K \backslash L) \leq n$.

Proof Case $1 L$ contains the 1-skeleton of $K$. Define $H: K \times \partial I \cup L \times I \rightarrow \mathbb{R} P^{n+1}$ in an obvious way (start from $f$, end at $g$, and put $H(x, t)=f(x)$ for $(x, t) \in L \times I)$. That way $H$ is defined on the $2-$ skeleton $(K \times I)^{(2)}$ of $K \times I$ after which one can extend it continuously over $K \times I$ due to $\pi_{m}\left(\mathbb{R} P^{n+1}\right)=0$ for $2 \leq m \leq n$.

Case $2 L$ contains the 0 -skeleton of $K$. Given an edge $e$ of $K$ that is not contained in $L$ we can create a homotopy from $f \mid e$ to $g \mid e$ relative to the vertices of $e$ as $\pi_{1}(f)=\pi_{1}(g)$. That means $f$ is homotopic rel. $L$ to a map $h$ such that $h \mid\left(L \cup K^{(1)}\right)=$ $g \mid\left(L \cup K^{(1)}\right)$. By Case $1, f$ is homotopic to $g$ rel. $L$.

General case Choose a maximal tree $T$ in the 1-skeleton of $K$. On each component $C$ of $T \backslash L$ we can create a homotopy from $f \mid \operatorname{cl}(C)$ to $g \mid \operatorname{cl}(C)$ relative to the 
boundary of $C$ as $\pi_{1}(f)=\pi_{1}(g)$. That means $f$ is homotopic rel. $L$ to a map $h$ such that $h|(L \cup T)=g|(L \cup T)$. By Case 2 (as $T$ contains all vertices of $K), f$ is homotopic to $g$ rel. $L$.

Corollary 2.2 Any two maps $u, v: \mathbb{R} P^{n} \rightarrow \mathbb{R} P^{n+1}, n \geq 2$, such that $u \mid \mathbb{R} P^{n-1}=$ $v \mid \mathbb{R} P^{n-1}$ are homotopic rel. $\mathbb{R} P^{n-1}$.

Corollary 2.3 Suppose $n \geq 0$. Given $u: \mathbb{R} P^{n} \times \mathbb{R} P^{1} \rightarrow \mathbb{R} P^{n+1}$ such that the restriction $u \mid \mathbb{R} P^{n} \times \mathbb{R} P^{0}$ is the inclusion and $u \mid \mathbb{R} P^{0} \times \mathbb{R} P^{1}$ determines a homotopically trivial loop in $\mathbb{R} P^{n+1}$, one can homotop $u$ rel. $\mathbb{R} P^{n} \times \mathbb{R} P^{0}$ to a map $v$ such that $v \mid \mathbb{R} P^{n} \times \mathbb{R} P^{0} \cup \mathbb{R} P^{n-1} \times \mathbb{R} P^{1}$ is the projection onto $\mathbb{R} P^{n}$ followed by the inclusion $\mathbb{R} P^{n} \rightarrow \mathbb{R} P^{n+1}$.

The map $v$ as in Corollary 2.3 will be called a normal form of $u$ and we shall say that $v$ is in normal form.

Similarly, given $n \geq 0$ and a map $u: \mathbb{R} P^{n} \times I \rightarrow \mathbb{R} P^{n+1}$ such that both $u_{0}$ and $u_{1}$ are inclusions and $u \mid \mathbb{R} P^{0} \times I$ determines a homotopically trivial loop in $\mathbb{R} P^{n+1}$, one can homotop $u$ rel. $\mathbb{R} P^{n} \times \partial I$ to a map $v$ : $\mathbb{R} P^{n} \times I \rightarrow \mathbb{R} P^{n+1}$ such that $v \mid \mathbb{R} P^{n-1} \times I$ is the projection onto $\mathbb{R} P^{n-1}$ followed by the inclusion $\mathbb{R} P^{n-1} \rightarrow \mathbb{R} P^{n+1}$. Again, we will call such $v$ a normal form of $u$, and $v$ is said to be in normal form.

Corollary 2.4 If $n \geq 1$ and $u, v: \mathbb{R} P^{n} \times \mathbb{R} P^{1} \rightarrow \mathbb{R} P^{n+1}$ are in normal form, then the following conditions are equivalent:

(a) $u$ and $v$ determine the same element of $\pi_{1}\left(\operatorname{Map}\left(\mathbb{R} P^{n}, \mathbb{R} P^{n+1}\right), i\right)$.

(b) $u$ and $v$ are homotopic rel. $\mathbb{R} P^{n} \times \mathbb{R} P^{0}$.

(c) $u$ and $v$ are homotopic rel. $\mathbb{R} P^{n} \times \mathbb{R} P^{0} \cup \mathbb{R} P^{n-2} \times \mathbb{R} P^{1}$.

Proof Obviously, (a) $\Leftrightarrow$ (b) and (c) $\Rightarrow$ (b), so it suffices to show (b) $\Rightarrow$ (c) if $n \geq 2$. Any homotopy from $u$ to $v$ rel. $\mathbb{R} P^{n} \times \mathbb{R} P^{0}$ induces $H: \mathbb{R} P^{n-2} \times \mathbb{R} P^{1} \times S^{1} \rightarrow \mathbb{R} P^{n+1}$ that is homotopically trivial on $\mathbb{R} P^{0} \times \mathbb{R} P^{0} \times S^{1}$. Using Lemma 2.1 we may accomplish $H$ to be the projection on the first coordinate followed by inclusion $\mathbb{R} P^{n-2} \rightarrow \mathbb{R} P^{n+1}$. $\square$

Suppose $v: \mathbb{R} P^{n} \times I \rightarrow \mathbb{R} P^{n+1}$ is in normal form for some $n \geq 1$. Let $B^{n}$ be the upper hemisphere of $S^{n} \subset S^{n+1}$. Consider $p_{n} \times$ id: $B^{n} \times I \rightarrow \mathbb{R} P^{n} \times I$ and let $\widetilde{v}: B^{n} \times I \rightarrow S^{n+1}$ be the lift

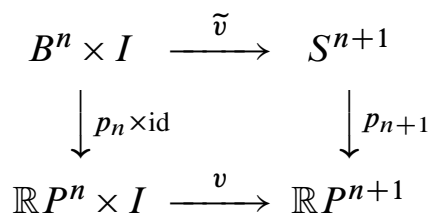


of $v \circ p_{n} \times$ id so that $\widetilde{v}_{0}$ is the inclusion $B^{n} \rightarrow S^{n+1}$. Notice $\widetilde{v}_{1}$ is also the inclusion and $\widetilde{v} \mid S^{n-1} \times I$ is the projection to $S^{n-1}$. Thus $\widetilde{v}\left(\partial\left(B^{n} \times I\right)\right) \subset B^{n}$ and we can talk about the degree $\operatorname{deg}(\widetilde{v})$ of the induced map $\left(B^{n} \times I\right) /\left(\partial\left(B^{n} \times I\right)\right) \rightarrow S^{n+1} / B^{n}$ once the orientations are fixed. Since we are only interested in the parity of the degree, the choice of orientations is irrelevant.

The above definition allows one to create the concept of the degree of any loop based at the inclusion in the space $\operatorname{Map}\left(\mathbb{R} P^{n}, \mathbb{R} P^{n+1}\right)$ of maps from $\mathbb{R} P^{n}$ to $\mathbb{R} P^{n+1}$ that is in normal form. That degree should not be confused with the group-theoretic degree of the element of $\pi_{1}\left(\operatorname{Map}\left(\mathbb{R} P^{n}, \mathbb{R} P^{n+1}\right), i\right.$ ) induced by the loop. Notice the degree of the concatenation of two loops equals the sum of degrees of the terms of concatenation. Lemma 2.5 below says that loops of even degree are null-homotopic in $\operatorname{Map}\left(\mathbb{R} P^{n}, \mathbb{R} P^{n+1}\right)$.

Lemma 2.5 Assume $\mathbb{R} P^{n-2} \subset \mathbb{R} P^{n-1} \subset \mathbb{R} P^{n}$ and $v: \mathbb{R} P^{n} \times I \rightarrow \mathbb{R} P^{n+1}$ is in normal form for some $n \geq 1$. $v$ is homotopic rel. $\mathbb{R} P^{n} \times \partial I \cup \mathbb{R} P^{n-2} \times I$ to the projection $\mathbb{R} P^{n} \times I \rightarrow \mathbb{R} P^{n}$ followed by inclusion if and only if $\operatorname{deg}(\widetilde{v})$ is even.

Proof Suppose $\operatorname{deg}(\widetilde{v})=2 k$. Express $S^{n-1}$ as the union $H_{+} \cup H_{-}$of upper and lower hemispheres.

Our plan is to find a homotopy $G: I \times B^{n} \times I \rightarrow S^{n+1}$ (its parameters come from the first $I$-coordinate) from $G_{0}=\tilde{v}$ to $G_{1}$ being the projection onto the second coordinate so that $p_{n+1} \circ G$ induces a homotopy on $\mathbb{R} P^{n} \times I$ as desired. That is accomplished by defining $G$ on $\partial\left(I \times B^{n} \times I\right)$ so that its degree is trivial which allows for a continuous extension of $G$ over the entire $I \times B^{n} \times I$.

Note that it follows from the homotopy constraints that the maps $G \mid \partial\left(I \times H_{+} \times I\right)$, $G\left|\partial\left(I \times H_{-} \times I\right), G^{0}=G\right|\left(I \times B^{n} \times 0\right)$ and $G^{1}=G \mid\left(I \times B^{n} \times 1\right)$ are defined as the projections to the second coordinate. Since $G_{0}$ and $G_{1}$ are known as well the only missing parts are $G_{+}=G \mid\left(I \times H_{+} \times I\right)$ and $G_{-}=G \mid\left(I \times H_{-} \times I\right)$. In order to induce a homotopy on $\mathbb{R} P^{n} \times I$ the map $G$ has to satisfy $G(s, x, t)=-G(s,-x, t)$ for $x \in S^{n-1}$ and hence $G_{-}$is determined by $G_{+}$.

Recall that the degree of the map $G_{0}:\left(0 \times B^{n} \times I, \partial\left(0 \times B^{n} \times I\right)\right) \rightarrow\left(S^{n+1}, B^{n}\right)$ is $2 k$ and note that the degrees of the maps $G_{1}:\left(1 \times B^{n} \times I, \partial\left(1 \times B^{n} \times I\right)\right) \rightarrow\left(S^{n+1}, B^{n}\right)$, $G^{0}:\left(I \times B^{n} \times 0, \partial\left(I \times B^{n} \times 0\right)\right) \rightarrow\left(S^{n+1}, B^{n}\right)$ and $G^{1}:\left(I \times B^{n} \times 1, \partial\left(I \times B^{n} \times 1\right)\right) \rightarrow$ $\left(S^{n+1}, B^{n}\right)$ are 0 (we fix orientations of $\partial\left(I \times B^{n} \times I\right)$ and $S^{n+1}$, and all the degrees are determined with respect to these orientations). Extend $G \mid \partial\left(I \times H_{+} \times I\right)$ to a map $G_{+}:\left(I \times H_{+} \times I, \partial\left(I \times H_{+} \times I\right)\right) \rightarrow\left(S^{n+1}, H_{+}\right)$of degree $-k$. Define the induced map $G_{-}:\left(I \times H_{-} \times I, \partial\left(I \times H_{-} \times I\right)\right) \rightarrow\left(S^{n+1}, H_{-}\right)$by $G_{-}(s, x, t)=$ 
$-G_{+}(s,-x, t)$ for $x \in H_{-}$. Since the degree of $\left(I \times H_{-} \times I, \partial\left(I \times H_{-} \times I\right)\right) \rightarrow$ $\left(I \times H_{+} \times I, \partial\left(I \times H_{+} \times I\right)\right)$ defined by $(s, x, t) \rightarrow(s,-x, t)$ is $(-1)^{n}$ and the degree of the map $\left(S^{n+1}, H_{+}\right) \rightarrow\left(S^{n+1}, H_{-}\right)$defined by $x \rightarrow-x$ is $(-1)^{n+2}$ we get that the degree of $G_{-}$is $(-1)^{n}(-k)(-1)^{n+2}=-k$. Note that the degrees of the inclusions $\left(S^{n+1}, H_{+}\right) \rightarrow\left(S^{n+1}, B^{n}\right)$ and $\left(S^{n+1}, H_{-}\right) \rightarrow\left(S^{n+1}, B^{n}\right)$ are 1. Then $G_{+}$and $G_{-}$considered as maps to $\left(S^{n+1}, B^{n}\right)$ are of degree $-k$. Thus the total degree of the map $G: \partial\left(I \times B^{n} \times I\right) \rightarrow S^{n+1}$ followed by the quotient map $S^{n+1} \rightarrow S^{n+1} / B^{n}$ is $2 k+(-k)+(-k)=0$ and hence so is the degree of $G$ and it can be extended continuously over $I \times B^{n} \times I$. Since $x \in S^{n-1}$ implies $G(s,-x, t)=-G(s, x, t), G$ indeed induces the desired homotopy between $v$ and the projection $\mathbb{R} P^{n} \times I \rightarrow \mathbb{R} P^{n+1}$.

Let us turn to the "only if" part of the proof. Assume that there is a homotopy $G$ with the required properties. Using the notation and the arguments of the previous part we deduce that the degree of $G$ restricted to $\partial\left(I \times B^{n} \times I\right)$ is $\operatorname{deg} G_{0}+2 \operatorname{deg} G_{+}$, where $G_{0}$ and $G_{+}$are considered as maps of the corresponding pairs to $\left(S^{n+1}, B^{n}\right)$. Thus we get that $\operatorname{deg} G_{0}+2 \operatorname{deg} G_{+}=\operatorname{deg} \tilde{v}+2 \operatorname{deg} G_{+}=0$ and we are done.

Theorem 2.6 Consider the inclusion $i: \mathbb{R} P^{n} \rightarrow \mathbb{R} P^{n+1}$. If $n \geq 1$, then the homomorphism

$$
\lambda: \pi_{1}\left(\operatorname{Map}\left(\mathbb{R} P^{n}, \mathbb{R} P^{n+1}\right), i\right) \rightarrow \mathbf{Z}_{2}
$$

is an epimorphism and its kernel is $\mathbf{Z}_{2}$.

Proof As $\mathbb{R} P^{\infty}$ is an Eilenberg-Mac Lane space $K\left(\mathbf{Z}_{2}, 1\right)$ there is a pointed cellular map $m: \mathbb{R} P^{\infty} \times \mathbb{R} P^{\infty} \rightarrow \mathbb{R} P^{\infty}$ corresponding to addition $\mathbf{Z}_{2} \times \mathbf{Z}_{2} \rightarrow \mathbf{Z}_{2}$ on the level of fundamental groups. Since $m\left(\mathbb{R} P^{n} \times \mathbb{R} P^{1}\right) \subset \mathbb{R} P^{n+1}$, the restriction $m \mid\left(\mathbb{R} P^{n} \times \mathbb{R} P^{1}\right)$ of $m$ induces a loop in $\left(\operatorname{Map}\left(\mathbb{R} P^{n}, \mathbb{R} P^{n+1}\right), i\right)$ whose evaluation is a nontrivial loop in $\mathbb{R} P^{n+1}$. That proves that $\lambda$ is an epimorphism.

Lemma 2.5 says that there are at most two homotopy classes of such maps depending on whether the degree of $v$ is even or not. To detect a nontrivial element of the kernel (see Part b) of Proposition 3.5 for a geometrical way of doing so in case $n \geq 2$ ) pick any $v$ in normal form so that the degree of its lift $\tilde{v}$

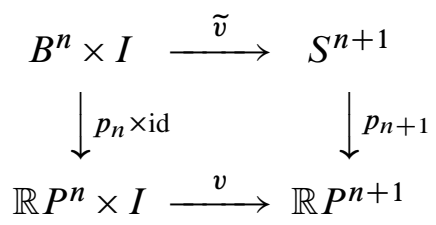

is 1 . A way to construct $v$ is by defining $\widetilde{v}: B^{n} \times I \rightarrow S^{n+1}$ first: $\widetilde{v}$ restricted to $\partial\left(B^{n} \times I\right)$ is the projection onto the first coordinate and then we extend it continuously 
over $B^{n} \times I$ so that the degree of the resulting map from $\left(B^{n} \times I\right) / \partial\left(B^{n} \times I\right) \rightarrow$ $S^{n+1} / B^{n}$ is 1 .

In view of Theorem 2.6 there are only two possibilities for the group (given $n \geq 1$ ) $\pi_{1}\left(\operatorname{Map}\left(\mathbb{R} P^{n}, \mathbb{R} P^{n+1}\right), i\right): \mathbf{Z}_{4}$ or $\mathbf{Z}_{2} \oplus \mathbf{Z}_{2}$. We do not know which case holds for a particular $n$, only for some initial values of $n$.

Proposition 2.7 If $n \geq 1$, then the following conditions are equivalent:

(a) $\pi_{1}\left(\operatorname{Map}\left(\mathbb{R} P^{n}, \mathbb{R} P^{n+1}\right), i\right)=\mathbf{Z}_{2} \oplus \mathbf{Z}_{2}$.

(b) There is $u$ : $\mathbb{R} P^{n} \times \mathbb{R} P^{2} \rightarrow \mathbb{R} P^{n+1}$ such that $u \mid\left(\mathbb{R} P^{n} \times \mathbb{R} P^{0}\right)$ is the projection to $\mathbb{R} P^{n}$ followed by the inclusion $\mathbb{R} P^{n} \rightarrow \mathbb{R} P^{n+1}$ and $u \mid\left(\mathbb{R} P^{0} \times \mathbb{R} P^{1}\right)$ is homotopically nontrivial.

Proof (a) $\Rightarrow$ (b) Pick a map $u: \mathbb{R} P^{n} \times \mathbb{R} P^{1} \rightarrow \mathbb{R} P^{n+1}$ which represents a loop in $\left(\operatorname{Map}\left(\mathbb{R} P^{n}, \mathbb{R} P^{n+1}\right), i\right)$ and does not belong to the kernel of $\lambda$. We may assume $u \mid \mathbb{R} P^{0} \times \mathbb{R} P^{1}$ is the inclusion. Since $u$ is of group-theoretic degree 2 , it extends continuously over $\mathbb{R} P^{n} \times \mathbb{R} P^{2}$.

(b) $\Rightarrow$ (a) Any such $u$ induces an element of order 2 not belonging to the kernel of $\lambda$. That means $\pi_{1}\left(\operatorname{Map}\left(\mathbb{R} P^{n}, \mathbb{R} P^{n+1}\right), i\right)$ cannot be $\mathbf{Z}_{4}$.

Corollary $2.8 \pi_{1}\left(\operatorname{Map}\left(\mathbb{R} P^{n}, \mathbb{R} P^{n+1}\right), i\right)=\mathbf{Z}_{2} \oplus \mathbf{Z}_{2}$ for $n=2,3,6,7$.

Proof The quaternionic multiplication on $S^{3} \times S^{3}$ induces a map $u: \mathbb{R} P^{3} \times \mathbb{R} P^{3} \rightarrow$ $\mathbb{R} P^{3}$ such that both $u \mid\left(\mathbb{R} P^{3} \times \mathbb{R} P^{0}\right)$ and $u \mid\left(\mathbb{R} P^{0} \times \mathbb{R} P^{3}\right)$ are inclusions. This takes care of $n=2,3$.

Using octonions (the Cayley numbers) and the multiplication $S^{7} \times S^{7} \rightarrow S^{7}$ one induces a map $u: \mathbb{R} P^{7} \times \mathbb{R} P^{7} \rightarrow \mathbb{R} P^{7}$ which handles $n=6,7$.

Corollary $2.9 \pi_{1}\left(\operatorname{Map}\left(\mathbb{R} P^{1}, \mathbb{R} P^{2}\right), i\right)=\mathbf{Z}_{4}$.

Proof If $\pi_{1}\left(\operatorname{Map}\left(\mathbb{R} P^{1}, \mathbb{R} P^{2}\right), i\right)=\mathbf{Z}_{2} \oplus \mathbf{Z}_{2}$, then Proposition 2.7(b) implies the existence of a map $u: \mathbb{R} P^{1} \times \mathbb{R} P^{2} \rightarrow \mathbb{R} P^{2}$ such that $u \mid\left(\mathbb{R} P^{1} \times \mathbb{R} P^{0}\right)$ and $u \mid\left(\mathbb{R} P^{0} \times \mathbb{R} P^{1}\right)$ are inclusions (any map from $\mathbb{R} P^{1}$ to $\mathbb{R} P^{2}$ that is not null-homotopic is homotopic to the inclusion - apply Lemma 2.1). Converting $u$ to $v: I \times \mathbb{R} P^{2} \rightarrow \mathbb{R} P^{2}$ and lifting it to $\widetilde{v}: I \times S^{2} \rightarrow S^{2}$ produces a homotopy joining a map $f$ on $S^{2}$ with the composition of itself and the antipodal map. That means $f$ must be homotopic to a constant map and we are led to a map $g: \mathbb{R} P^{2} \rightarrow \mathbb{R} P^{2}$ (induced by $v \mid\left(\{0\} \times \mathbb{R} P^{2}\right)$ ) inducing a nontrivial homomorphism on the fundamental group but $\pi_{2}(g)$ is trivial. Hence $g$ can 
be extended continuously over $\mathbb{R} P^{3}$ (look at the attaching map of the 3-cell of $\mathbb{R} P^{3}$ ) contradicting Borsuk-Ulam Theorem (any continuous function from the $n$-sphere into Euclidean $n$-space maps some pair of antipodal points to the same point). Indeed, the lift $h: S^{3} \rightarrow S^{2} \subset R^{3}$ of $g: \mathbb{R} P^{3} \rightarrow \mathbb{R} P^{2}$ must satisfy $g(-x)=-g(x)$ for all $x \in S^{3}$ (otherwise $\pi_{1}(g)=0$ ).

\section{Dimension reduction via tubular neighborhoods}

In this section we are interested in altering maps to $\mathbb{R} P^{n+2}$ into maps to $\mathbb{R} P^{n+1}$ relative to $\mathbb{R} P^{2}$ (see Corollary 3.6). That is done by pushing a map off a part of a closed tubular neighborhood of the perpendicular $\mathbb{R} P_{\perp}^{n}$ to $\mathbb{R} P^{2}$ in $\mathbb{R} P^{n+2}$.

Definition 3.1 Given a closed subset $L$ of a space $K$ by $(K \times F) /(L \times F)$ we mean the quotient space of $K \times F$ under the decomposition consisting of singletons $(x, y) \notin L \times F$ and sets $\{x\} \times F$ for $x \in L$. Notice one has a natural projection $\pi_{K}:(K \times F) /(L \times F) \rightarrow K$.

Alternatively, $(K \times F) /(L \times F)$ is the adjunction space $(K \times F) \cup_{\pi} L$, where $\pi: L \times F \rightarrow F$ is the projection. In particular, if $F$ is a finite $\mathrm{CW}-$ complex and $L$ is a subcomplex of a CW-complex $K$, then $(K \times F) /(L \times F)$ is a CW-complex.

The main and defining property of $\pi_{K}$ is that the projection $\operatorname{proj}_{K}: K \times F \rightarrow K$ factors as $q \circ \pi_{K}$, where $q: K \times F \rightarrow(K \times F) /(L \times F)$ is the quotient map. The continuity of $\pi_{K}$ follows from the fact that $q$ is a quotient map.

Of primary interest are the spaces $\left(\mathbb{R} P^{n} \times \mathbb{R} P^{1}\right) /\left(\mathbb{R} P^{n-2} \times \mathbb{R} P^{1}\right)$, for $n>1$. For such a space, the 2 -skeleton coincides with the 2-skeleton of the union $\mathbb{R} P^{n} \times$ $\mathbb{R} P^{0} \cup\left(\mathbb{R} P^{1} \times \mathbb{R} P^{1}\right) /\left(\mathbb{R} P^{0} \times \mathbb{R} P^{1}\right)$, therefore the inclusion of $\mathbb{R} P^{n} \times \mathbb{R} P^{0}$ into $\left(\mathbb{R} P^{n} \times \mathbb{R} P^{1}\right) /\left(\mathbb{R} P^{n-2} \times \mathbb{R} P^{1}\right)$ induces an isomorphism of the fundamental groups.

It turns out there are precisely (up to homotopy) two maps

$$
s: \frac{\mathbb{R} P^{n} \times \mathbb{R} P^{1}}{\mathbb{R} P^{n-2} \times \mathbb{R} P^{1}} \rightarrow \mathbb{R} P^{n+1}
$$

that induce a nontrivial homomorphism of the fundamental groups. We will show this by displaying a connection between such maps and the kernel of

$$
\lambda: \pi_{1}\left(\operatorname{Map}\left(\mathbb{R} P^{n}, \mathbb{R} P^{n+1}\right), i\right) \rightarrow \mathbf{Z}_{2} .
$$

For the map $u$ : $\mathbb{R} P^{n} \times \mathbb{R} P^{1} \rightarrow \mathbb{R} P^{n+1}$ to be in normal form means precisely that it factors as $\mathbb{R} P^{n} \times \mathbb{R} P^{1} \rightarrow\left(\mathbb{R} P^{n} \times \mathbb{R} P^{1}\right) /\left(\mathbb{R} P^{n-1} \times \mathbb{R} P^{1}\right) \rightarrow \mathbb{R} P^{n+1}$ (the first map 
being the natural projection) and $u \mid\left(\mathbb{R} P^{n} \times \mathbb{R} P^{0}\right)$ is the projection to $\mathbb{R} P^{n}$ followed by inclusion to $\mathbb{R} P^{n+1}$. If $n \geq 2$, then any $u$ : $\mathbb{R} P^{n} \times \mathbb{R} P^{1} \rightarrow \mathbb{R} P^{n+1}$ in normal form induces $\widehat{u}$ : $\left(\mathbb{R} P^{n} \times \mathbb{R} P^{1}\right) /\left(\mathbb{R} P^{n-2} \times \mathbb{R} P^{1}\right) \rightarrow \mathbb{R} P^{n+1}$. Namely, $\hat{u}$ arises from factorization of $u$ as $\mathbb{R} P^{n} \times \mathbb{R} P^{1} \rightarrow\left(\mathbb{R} P^{n} \times \mathbb{R} P^{1}\right) /\left(\mathbb{R} P^{n-2} \times \mathbb{R} P^{1}\right) \rightarrow \mathbb{R} P^{n+1}$. Therefore $\pi_{1}(\widehat{u})$ is surjective (as $\pi_{1}(u)$ is surjective). The reverse statement is less obvious.

Proposition 3.2 Suppose $n \geq 2$. If $s:\left(\mathbb{R} P^{n} \times \mathbb{R} P^{1}\right) /\left(\mathbb{R} P^{n-2} \times \mathbb{R} P^{1}\right) \rightarrow \mathbb{R} P^{n+1}$ is a map and $\pi_{1}(s)$ is nontrivial, then there is $u: \mathbb{R} P^{n} \times \mathbb{R} P^{1} \rightarrow \mathbb{R} P^{n+1}$ in normal form such that $\hat{u}$ is homotopic to $s$.

Proof Consider the restriction $w$ of $s$ to $K=\left(\mathbb{R} P^{n-1} \times \mathbb{R} P^{1}\right) /\left(\mathbb{R} P^{n-2} \times \mathbb{R} P^{1}\right)$ and let $p:\left(\mathbb{R} P^{n-1} \times \mathbb{R} P^{1}\right) /\left(\mathbb{R} P^{n-2} \times \mathbb{R} P^{1}\right) \rightarrow \mathbb{R} P^{n+1}$ be the projection onto $\mathbb{R} P^{n-1}$ followed by inclusion $\mathbb{R} P^{n-1} \rightarrow \mathbb{R} P^{n+1}$. Both restrictions $s \mid\left(\mathbb{R} P^{n-1} \times \mathbb{R} P^{0}\right)$ and $p \mid\left(\mathbb{R} P^{n-1} \times \mathbb{R} P^{0}\right)$ must be homotopic by Corollary 2.2 , so applying Lemma 2.1 results in $s$ being homotopic to $p$. So assume $s$ equals $p$. Now $s \mid \mathbb{R} P^{n} \times \mathbb{R} P^{0}$ is homotopic to the inclusion rel. $\mathbb{R} P^{n-1} \times \mathbb{R} P^{0}$ (see Corollary 2.2), so we may assume $s$ restricted to $\mathbb{R} P^{n} \times \mathbb{R} P^{0} \cup\left(\mathbb{R} P^{n-1} \times \mathbb{R} P^{1}\right) /\left(\mathbb{R} P^{n-2} \times \mathbb{R} P^{1}\right)$ is the projection onto the first coordinate. That is equivalent to the composition $u$ of

$$
\mathbb{R} P^{n} \times \mathbb{R} P^{1} \stackrel{\text { proj }}{\rightarrow} \frac{\mathbb{R} P^{n} \times \mathbb{R} P^{1}}{\mathbb{R} P^{n-2} \times \mathbb{R} P^{1}} \stackrel{s}{\rightarrow} \mathbb{R} P^{n+1}
$$

being in normal form and $s=\widehat{u}$.

Corollary 3.3 Let $n \geq 2$. If $\pi_{1}(s)$ of $s:\left(\mathbb{R} P^{n} \times \mathbb{R} P^{1}\right) /\left(\mathbb{R} P^{n-2} \times \mathbb{R} P^{1}\right) \rightarrow \mathbb{R} P^{n+1}$ is nontrivial, then $s$ extends continuously over $\left(\mathbb{R} P^{n} \times \mathbb{R} P^{2}\right) /\left(\mathbb{R} P^{n-2} \times \mathbb{R} P^{2}\right)$.

Proof From Proposition 3.2 it suffices to consider the case of the composition $u$ of

$$
\mathbb{R} P^{n} \times \mathbb{R} P^{1} \stackrel{\text { proj }}{\rightarrow} \frac{\mathbb{R} P^{n} \times \mathbb{R} P^{1}}{\mathbb{R} P^{n-2} \times \mathbb{R} P^{1}} \stackrel{s}{\rightarrow} \mathbb{R} P^{n+1}
$$

being in normal form (thus $s=\widehat{u}$ ). The composition $v$ of

$$
\mathbb{R} P^{n} \times S^{1} \stackrel{\mathrm{id} \times p_{1}}{\longrightarrow} \mathbb{R} P^{n} \times \mathbb{R} P^{1} \stackrel{u}{\rightarrow} \mathbb{R} P^{n+1}
$$

induces an element of $\pi_{1}\left(\operatorname{Map}\left(\mathbb{R} P^{n}, \mathbb{R} P^{n+1}\right), i\right)$ that becomes trivial under the homomorphism $\lambda$. According to Theorem 2.6 that element is of group-theoretic degree two (in $\pi_{1}\left(\operatorname{Map}\left(\mathbb{R} P^{n}, \mathbb{R} P^{n+1}\right)\right)$ ), so it extends continuously over $\mathbb{R} P^{n} \times \mathbb{R} P^{2}$. Let $G: \mathbb{R} P^{n} \times \mathbb{R} P^{2} \rightarrow \mathbb{R} P^{n+1}$ be such extension. By Corollary $2.2 G$ restricted to $\mathbb{R} P^{n-2} \times \mathbb{R} P^{2}$ is homotopic rel. $\mathbb{R} P^{n-2} \times \mathbb{R} P^{1}$ to the projection onto the first coordinate. That demonstrates the existence of a map $F:\left(\mathbb{R} P^{n} \times \mathbb{R} P^{2}\right) /\left(\mathbb{R} P^{n-2} \times \mathbb{R} P^{2}\right) \rightarrow$ $\mathbb{R} P^{n+1}$ whose restriction to $\left(\mathbb{R} P^{n} \times \mathbb{R} P^{1}\right) /\left(\mathbb{R} P^{n-2} \times \mathbb{R} P^{1}\right)$ is homotopic to $s$. 
Let $N$ be a closed tubular neighborhood of $\mathbb{R} P^{n}$ in $\mathbb{R} P^{n+2}(n \geq 1)$ and let $\theta: \partial N \rightarrow$ $\mathbb{R} P^{n}$ be the corresponding circle bundle. The main property of $N$ we are interested in is that $\theta$ is trivial over $\mathbb{R} P^{n} \backslash \mathbb{R} P^{n-2}$ (note that $\theta$ is not trivial over $\mathbb{R} P^{n} \backslash \mathbb{R} P^{n-3}$ for $n \geq 2$ ).

Lemma 3.4 If $n \geq 2$, then $\mathbb{R} P^{n}$ has a closed tubular neighborhood in $\mathbb{R} P^{n+2}$ such that the corresponding circle bundle is trivial over $\mathbb{R} P^{n} \backslash \mathbb{R} P^{n-2}$.

Proof Represent $\mathbb{R} P^{n+2}$ as equivalence classes $\left[x_{1}, x_{2}, x_{3}, x_{4}, \ldots, x_{n+3}\right]$ obtained from identifying antipodal points in the unit $(n+2)$-sphere. Under this model $\mathbb{R} P^{n}$ is the set of points $\left[0,0, x_{3}, x_{4}, \ldots, x_{n+3}\right]$ and $\mathbb{R} P^{n} \backslash \mathbb{R} P^{n-2}$ is the set of points $\left[0,0, x_{3}, x_{4}, \ldots, x_{n+3}\right]$ such that $x_{3}^{2}+x_{4}^{2} \neq 0$. We will consider $N$ to be the set of points $\left[x_{1}, x_{2}, x_{3}, x_{4}, \ldots, x_{n+3}\right]$ such that $x_{1}^{2}+x_{2}^{2} \leq \frac{1}{9}$ and the disk bundle $\pi: N \rightarrow$ $\mathbb{R} P^{n}$ is given by $\pi\left(\left[x_{1}, x_{2}, x_{3}, x_{4}, \ldots, x_{n+3}\right]\right)=\left[0,0, t \cdot x_{3}, t \cdot x_{4}, \ldots, t \cdot x_{n+3}\right]$, where $t=\sqrt{1 /\left(1-x_{1}^{2}-x_{2}^{2}\right)}$. Let us show that the corresponding circle bundle $\theta: \partial N \rightarrow \mathbb{R} P^{n}$ is trivial over $\mathbb{R} P^{n} \backslash \mathbb{R} P^{n-2}$ by exhibiting two maps $\phi: \theta^{-1}\left(\mathbb{R} P^{n} \backslash \mathbb{R} P^{n-2}\right) \rightarrow$ $\left(\mathbb{R} P^{n} \backslash \mathbb{R} P^{n-2}\right) \times S^{1}$ and $\psi:\left(\mathbb{R} P^{n} \backslash \mathbb{R} P^{n-2}\right) \times S^{1} \rightarrow \theta^{-1}\left(\mathbb{R} P^{n} \backslash \mathbb{R} P^{n-2}\right)$ that are inverse to each other. $\phi$ is defined by the formula

$$
\phi\left(\left[x_{1}, x_{2}, x_{3}, x_{4}, \ldots, x_{n+3}\right]\right)=\left(\left[0,0, t \cdot x_{3}, t \cdot x_{4}, \ldots, t \cdot x_{n+3}\right], z /|z|\right)
$$

where $t=\sqrt{1 /\left(1-x_{1}^{2}-x_{2}^{2}\right)}=\sqrt{9 / 8}$ and $z=\left(x_{1}+i \cdot x_{2}\right) \cdot\left(x_{3}+i \cdot x_{4}\right) . \psi$ is defined by the formula

$$
\psi\left(\left[0,0, x_{3}, x_{4}, \ldots, x_{n+3}\right], w\right)=\left[x_{1}, x_{2}, s \cdot x_{3}, s \cdot x_{4}, \ldots, s \cdot x_{n+3}\right]
$$

where $s=\sqrt{8 / 9}$ and $x_{1}+i \cdot x_{2}=z /(3|z|), z=w /\left(x_{3}+i \cdot x_{4}\right)$.

Proposition 3.5 Consider a closed tubular neighborhood $N$ of $\mathbb{R} P^{n}$ in $\mathbb{R} P^{n+2}$ for some $n \geq 2$. Let $\theta: N \rightarrow \mathbb{R} P^{n}$ be the corresponding disk bundle and pick $x_{0} \in$ $\operatorname{Int}(N) \backslash D$ for some fiber $D=\theta^{-1}\left(x_{1}\right), x_{1} \in \mathbb{R} P^{n-2} \subset \mathbb{R} P^{n}$, of $\theta$.

(a) For any $\mathbb{R} P^{n+1} \subset \mathbb{R} P^{n+2} \backslash\left\{x_{0}\right\}$ the inclusion $\mathbb{R} P^{n+1} \rightarrow \mathbb{R} P^{n+2} \backslash\left\{x_{0}\right\}$ is a homotopy equivalence.

(b) The inclusion $i: \partial N \cup D \rightarrow \mathbb{R} P^{n+2} \backslash\left\{x_{0}\right\}$ factors up to homotopy as

$$
\partial N \cup D \rightarrow(\partial N \cup D) / D \rightarrow \frac{\mathbb{R} P^{n} \times \mathbb{R} P^{1}}{\mathbb{R} P^{n-2} \times \mathbb{R} P^{1}} \stackrel{s}{\rightarrow} \mathbb{R} P^{n+2} \backslash\left\{x_{0}\right\},
$$

where the composition

$$
\partial N \cup D \rightarrow(\partial N \cup D) / D \rightarrow \frac{\mathbb{R} P^{n} \times \mathbb{R} P^{1}}{\mathbb{R} P^{n-2} \times \mathbb{R} P^{1}}
$$


followed by the projection to the first coordinate coincides with $\theta$ restricted to $\partial N \cup D, s$ is not homotopic to the projection onto the first coordinate and $\pi_{1}(s)$ is nontrivial.

Proof (a) This is obvious from the representation of $\mathbb{R} P^{n+2}$ as the quotient of the $(n+2)$-ball $B^{n+2}$ : removing a point in the interior of $B^{n+2}$ allows for a deformation retraction to its boundary.

(b) Obviously, $i$ restricted to a fiber of $\theta$ is null-homotopic. On $M=\theta^{-1}\left(\mathbb{R} P^{n-2}\right)$ the map $\left.i\right|_{M}$ represents an element of $H^{1}\left(M ; \mathbf{Z}_{2}\right)$ as $\operatorname{dim}(M)=n-1$ and $\mathbb{R} P^{n+2} \backslash\left\{x_{0}\right\}$ is homotopy equivalent to some $\mathbb{R} P^{n+1}$ by a). So, by Proposition 5.1 it can be factored up to homotopy through $\mathbb{R} P^{n-2}$. Since $\theta$ is trivial over $\mathbb{R} P^{n} \backslash \mathbb{R} P^{n-2},\left.i\right|_{\partial N}$ factors up to homotopy as $s \circ p$, where $p: \partial N \rightarrow\left(\mathbb{R} P^{n} \times \mathbb{R} P^{1}\right) /\left(\mathbb{R} P^{n-2} \times \mathbb{R} P^{1}\right)$ sends fibers to fibers and $s:\left(\mathbb{R} P^{n} \times \mathbb{R} P^{1}\right) /\left(\mathbb{R} P^{n-2} \times \mathbb{R} P^{1}\right) \rightarrow \mathbb{R} P^{n+1}$ (see Proposition 5.2). Obviously, we can extend $p$ continuously over $D$ (by defining $p$ to be constant on $D)$ and the reason $s \circ p$ is homotopic to $i$ on $\partial N \cup D$ is that $\pi_{2}\left(\mathbb{R} P^{n+1}\right)=0$ as $n \geq 2$ (the original homotopy on $\partial N$ can be extended continuously over $D \times I$ ). If $s$ were homotopic to the projection onto the first coordinate, then the inclusion $\partial N \rightarrow \mathbb{R} P^{n+2} \backslash\left\{x_{0}\right\}$ could be factored up to homotopy (use Corollary 2.4) through the projection $\partial N \rightarrow \mathbb{R} P^{n}$ and would be continuously extendable over $N$. That would lead to a map $\mathbb{R} P^{n+2} \rightarrow \mathbb{R} P^{n+1}$ that is nontrivial on the fundamental group contradicting the Borsuk-Ulam Theorem (after lifting to the covering spheres). $\pi_{1}(s)$ is nontrivial as $\pi_{1}(i)$ is not trivial (a nontrivial loop in $\mathbb{R} P^{n+2}$ can be found in $\mathbb{R} P^{n} \backslash \mathbb{R} P^{n-2}$ and then pushed to $\partial N)$.

Corollary 3.6 Suppose $X$ is a metrizable space such that $X \tau \Sigma\left(\mathbb{R} P^{2}\right)$ and $n \geq 2$. If $X \tau\left(\mathbb{R} P^{2} \rightarrow \mathbb{R} P^{n+2}\right)$, then $X \tau\left(\mathbb{R} P^{2} \rightarrow \mathbb{R} P^{n+1}\right)$.

Proof Let $\mathbb{R} P^{2} \subset \mathbb{R} P^{n+1} \subset \mathbb{R} P^{n+2}$. Pick perpendicular $\mathbb{R} P^{n}$ to $\mathbb{R} P^{2}$ in $\mathbb{R} P^{n+2}$. Let $N$ be a closed tubular neighborhood of $\mathbb{R} P^{n}$ in $\mathbb{R} P^{n+2}$ and let $i: \partial N \rightarrow M=$ $\mathbb{R} P^{n+2} \backslash x_{0}$ be the inclusion where $x_{0} \in N \backslash\left(\partial N \cup \mathbb{R} P^{n+1}\right)$. Notice the inclusion $\mathbb{R} P^{n+1} \rightarrow M$ is a homotopy equivalence.

Given a closed subset $A$ of $X$ and a map $f: A \rightarrow \mathbb{R} P^{2}$ extend it continuously to a map $F: X \rightarrow \mathbb{R} P^{n+2}$. Let $D$ be the disk $\mathbb{R} P^{2} \cap N$ (we assume that $N$ and the corresponding disk bundle are chosen so that $D=\mathbb{R} P^{2} \cap N$ is a fiber of the bundle) and put $Y=F^{-1}(N), C=F^{-1}(\partial N \cup D)$. By Proposition 3.5 the inclusion $i: \partial N \cup D \rightarrow \mathbb{R} P^{n+2} \backslash\left\{x_{0}\right\}$ factors up to homotopy as the composition $\partial N \cup D \rightarrow(\partial N \cup D) / D \rightarrow\left(\mathbb{R} P^{n} \times \mathbb{R} P^{1}\right) /\left(\mathbb{R} P^{n-2} \times \mathbb{R} P^{1}\right) \stackrel{s}{\rightarrow} \mathbb{R} P^{n+2} \backslash$ $\left\{x_{0}\right\}$, where $s$ has properties described in Proposition 3.5(b) By Corollary 3.3, $s$ 
extends continuously over $\left(\mathbb{R} P^{n} \times \mathbb{R} P^{2}\right) /\left(\mathbb{R} P^{n-2} \times \mathbb{R} P^{2}\right)$, so we have maps $C \rightarrow$ $\partial N \cup D \rightarrow\left(\mathbb{R} P^{n} \times \mathbb{R} P^{2}\right) /\left(\mathbb{R} P^{n-2} \times \mathbb{R} P^{2}\right)$ whose composition with the projection $\left(\mathbb{R} P^{n} \times \mathbb{R} P^{2}\right) /\left(\mathbb{R} P^{n-2} \times \mathbb{R} P^{2}\right) \rightarrow \mathbb{R} P^{n}$ extends continuously over $Y$ (that extension is $F: Y \rightarrow N$ followed by the projection of $N$ onto $\left.\mathbb{R} P^{n}\right)$. By Corollary 5.4 there is $G: Y \rightarrow M$ agreeing with $f$ on $C$. Paste $G$ with $\left.F\right|_{X \backslash \operatorname{Int}(Y)}$ and get an extension of $f$ with values in $M$. As the inclusion $\mathbb{R} P^{n+1} \rightarrow M$ is a homotopy equivalence, the proof is completed.

\section{Main results}

Lemma 4.1 Let $a \in S^{1}$. Suppose $X$ is a metrizable space such that $X \tau \Sigma\left(\mathbb{R} P^{2}\right)$. Let $r_{i}: S^{1} \times \mathbb{R} P^{2} \rightarrow \mathbb{R} P^{2}, i=1,2$, be maps such that $r_{i} \mid a \times \mathbb{R} P^{1} \simeq$ const. If $A$ is closed in $X$ and $f: A \rightarrow S^{1} \times S^{1} \times \mathbb{R} P^{2}$ has the property that the composition

$$
A \stackrel{f}{\rightarrow} S^{1} \times S^{1} \times \mathbb{R} P^{2} \stackrel{\text { proj }}{\longrightarrow} S^{1} \times S^{1}
$$

extends continuously over $X$, then the composition

$$
A \stackrel{f}{\rightarrow} S^{1} \times S^{1} \times \mathbb{R} P^{2} \stackrel{\text { id } \times r_{1}}{\longrightarrow} S^{1} \times \mathbb{R} P^{2} \stackrel{r_{2}}{\longrightarrow} \mathbb{R} P^{2}
$$

extends continuously over $X$.

Proof Notice that any composition of two maps from $\mathbb{R} P^{2}$ to $\mathbb{R} P^{2}$ that are trivial on the fundamental group, is homotopically trivial. The reason is that each of them factors through $S^{2}$ and any composition $S^{2} \rightarrow \mathbb{R} P^{2} \rightarrow S^{2}$ is null-homotopic as it is of degree 0 . Thus the composition

$$
S^{1} \times S^{1} \times \mathbb{R} P^{2} \stackrel{\text { id } \times r_{1}}{\longrightarrow} S^{1} \times \mathbb{R} P^{2} \stackrel{r_{2}}{\longrightarrow} \mathbb{R} P^{2}
$$

is homotopically trivial on the fibers of the projection

$$
S^{1} \times S^{1} \times \mathbb{R} P^{2} \stackrel{\text { proj }}{\longrightarrow} S^{1} \times S^{1}
$$

and we can apply Corollary 5.5 directly.

In [10] we defined the second modification $M_{2}$ of $\mathbb{R} P^{3}$. In this paper we need its subset $M$ that we call the basic modification of $\mathbb{R} P^{3}$.

Definition 4.2 (Basic modification of $\mathbb{R} P^{3}$ ) Let $\mathbb{R} P^{1} \subset \mathbb{R} P^{2} \subset \mathbb{R} P^{3}$. Represent $\mathbb{R} P^{1}, \mathbb{R} P^{2}$ and $\mathbb{R} P^{3}$ as follows: $\mathbb{R} P^{3}$ is the quotient space of the unit ball in $R^{3}$, $\mathbb{R} P^{1}$ is the image of the unit circle in the $x y$-plane, and $\mathbb{R} P^{2}$ is the image of the unit 
sphere. Let $\mathbb{R} P_{*}^{1}$ be the image of the unit segment in the $z$-axis (it is the intersection of the $z$-axis and the unit ball $B^{3}$ ). Let $T$ be a closed tubular neighborhood of $\mathbb{R} P_{*}^{1}$ such that $T$ does not intersect $\mathbb{R} P^{1}$, and $x_{0} \in \operatorname{Int}(T) \backslash \mathbb{R} P^{2}$. Let $M=\mathbb{R} P^{3} \backslash \operatorname{Int}(T)$ and let $r_{M}: M \rightarrow \mathbb{R} P^{2}$ be the restriction to $M$ of a retraction $\mathbb{R} P^{3} \backslash\left\{x_{0}\right\} \rightarrow \mathbb{R} P^{2}$. Note that $T$ is a solid torus and can be represented as $T=S^{1} \times D$ with $D$ being a disk. Also note that $r_{M}$ restricted to $a \times \partial D$ is homotopically trivial and $r_{M}$ restricted to $S^{1} \times b$ is essential.

Lemma 4.3 Suppose $X$ is a metrizable space such that $X \tau \Sigma\left(\mathbb{R} P^{2}\right)$ and $X \tau\left(\mathbb{R} P^{1} \rightarrow\right.$ $\left.\mathbb{R} P^{3}\right)$. Let $r: S^{1} \times S^{1} \rightarrow \mathbb{R} P^{2}$ be a map such that $r \mid a \times S^{1} \simeq$ const and $r \mid S^{1} \times b$ is not null-homotopic. If $A$ is closed in $X$ and a map $f: A \rightarrow S^{1} \times S^{1}$ has the property that

$$
A \stackrel{f}{\rightarrow} S^{1} \times S^{1} \stackrel{\text { proj }_{1}}{\longrightarrow} S^{1}
$$

extends continuously over $X$, then

$$
A \stackrel{f}{\rightarrow} S^{1} \times S^{1} \stackrel{r}{\rightarrow} \mathbb{R} P^{2}
$$

extends continuously over $X$.

Proof Throughout the proof we treat $\partial D, S^{1}$ and $\mathbb{R} P^{1}$ interchangeably each time it is suggested by the context.

Without loss of generality we may assume $r \mid a \times S^{1}=$ const and use 2.4 of [10] to extend $r$ continuously to $r: S^{1} \times \mathbb{R} P^{2} \rightarrow \mathbb{R} P^{2}$.

Continuously extend the composition

$$
A \stackrel{f}{\rightarrow} S^{1} \times S^{1} \stackrel{\text { proj }_{2}}{\longrightarrow} S^{1} \stackrel{\text { inclusion }}{\longrightarrow} \mathbb{R} P^{3}
$$

over $X$. Let $g: X \rightarrow \mathbb{R} P^{3}$ be such extension. That means we have an extension $F: X \rightarrow S^{1} \times \mathbb{R} P^{3}$ of

$$
A \stackrel{f}{\rightarrow} S^{1} \times S^{1} \stackrel{\text { inclusion }}{\longrightarrow} S^{1} \times \mathbb{R} P^{3} .
$$

Let $T=S^{1} \times D$ be the solid torus in $\mathbb{R} P^{3}$ arising from the basic modification $M$ of $\mathbb{R} P^{3}$ (see Definition 4.2). Let $\left(f_{1}, f_{2}\right)$ be the coordinate maps of $f$. Then $F=$ $\left(f_{1}, g\right)$. Put $Y=F^{-1}\left(S^{1} \times T\right)=g^{-1}(T)$ and $C=F^{-1}\left(S^{1} \times \partial T\right)=g^{-1}(\partial T)$, $Z=F^{-1}\left(S^{1} \times M\right)=g^{-1}(M)$. By 2.4 of [10], $r_{M}$ restricted to $\partial T=S^{1} \times \partial D$ extends continuously to a map $r^{\prime}: S^{1} \times \mathbb{R} P^{2} \rightarrow \mathbb{R} P^{2}$ (see also Corollary 3.3). By Lemma 4.1 the composition

$$
C \stackrel{f_{1} \times g}{\rightarrow} S^{1} \times S^{1} \times \partial D \stackrel{\text { inclusion }}{\longrightarrow} S^{1} \times S^{1} \times \mathbb{R} P^{2} \stackrel{\text { id } \times r^{\prime}}{\longrightarrow} S^{1} \times \mathbb{R} P^{2} \stackrel{r}{\rightarrow} \mathbb{R} P^{2}
$$


extends continuously over $Y$. On $Z=g^{-1}\left(\mathbb{R} P^{3} \backslash \operatorname{Int}(T)\right)$ we have the map

$$
Z \rightarrow S^{1} \times M \stackrel{\operatorname{id} \times r_{M}}{\longrightarrow} S^{1} \times \mathbb{R} P_{2} \stackrel{r}{\rightarrow} \mathbb{R} P^{2}
$$

that can be pasted with the one on $Y$ to yield the desired $X \rightarrow \mathbb{R} P^{2}$.

Theorem 4.4 Suppose $X$ is a metrizable space. If $X \tau\left(\mathbb{R} P^{1} \rightarrow \mathbb{R} P^{2}\right)$ and $X \tau\left(\mathbb{R} P^{2} \rightarrow\right.$ $\mathbb{R} P^{3}$ ), then $X \tau \mathbb{R} P^{2}$.

Proof Suppose $f: A \rightarrow \mathbb{R} P^{2}, A$ being closed in $X$. Extend $f$ continuously to a map $\tilde{f}: X \rightarrow \mathbb{R} P^{3}$. Represent $\mathbb{R} P^{3}$ as the quotient of $B^{3}$ with $\mathbb{R} P^{2}$ being the image of $S^{2}=\partial B^{3}$. Remove the interior of an unknotted solid torus $T=S^{1} \times D$ in the interior of $B^{3}$. Attach $S^{1} \times \mathbb{R} P^{2}$ to $B^{3} \backslash \operatorname{Int}(T)$ via the identity on $S^{1}$ times a homeomorphism $\partial D \rightarrow \mathbb{R} P^{1}$. The resulting space $M$ retracts onto $\mathbb{R} P^{2}$ as shown in [10] (it is called the first modification of $\mathbb{R} P^{3}$ there). Put $C=\tilde{f}^{-1}(T)$ and $E=\tilde{f}^{-1}(\partial T)$. Since $C \tau\left(\mathbb{R} P^{1} \rightarrow \mathbb{R} P^{2}\right),\left.\tilde{f}\right|_{E}: E \rightarrow \partial T$ extends continuously to $G: C \rightarrow S^{1} \times \mathbb{R} P^{2}$. Replace $\tilde{f}$ by $G$ on $C$ and follow the retraction of $M$ onto $\mathbb{R} P^{2}$ to get an extension of $f$ from $X$ to $\mathbb{R} P^{2}$.

Theorem 4.5 Suppose $X$ is a metrizable space such that $X \tau \Sigma\left(\mathbb{R} P^{2}\right)$. If $X \tau\left(\mathbb{R} P^{1} \rightarrow\right.$ $\left.\mathbb{R} P^{3}\right)$, then $X \tau\left(\mathbb{R} P^{1} \rightarrow \mathbb{R} P^{2}\right)$.

Proof Given a map $f: A \rightarrow \mathbb{R} P^{1}$ and its continuous extension $F: X \rightarrow \mathbb{R} P^{3}$ put $Y=F^{-1}(T), T=S^{1} \times D$ being the solid torus arising from the basic modification $M$ of $\mathbb{R} P^{3}$ (see Definition 4.2). Since

$$
F^{-1}(\partial T) \rightarrow S^{1} \times \partial D \stackrel{r_{M}}{\longrightarrow} \mathbb{R} P^{2}
$$

extends continuously over $Y$ to $g: Y \rightarrow \mathbb{R} P^{2}$ by Lemma 4.3, one can paste $g$ with $F$ restricted to $X \backslash F^{-1}(\operatorname{Int}(T))$ followed by $r_{M}$ to obtain a continuous extension $X \rightarrow M$ of $f$.

Corollary 4.6 Suppose $X$ is a metrizable space. If $X \tau \Sigma\left(\mathbb{R} P^{2}\right)$, then the following conditions are equivalent:

(1) $X \tau \mathbb{R} P^{2}$

(2) $X \tau\left(\mathbb{R} P^{2} \rightarrow \mathbb{R} P^{3}\right)$

(3) $X \tau\left(\mathbb{R} P^{2} \rightarrow \mathbb{R} P^{n}\right)$ for some $n \geq 3$

Proof It suffices to prove (2) $\Rightarrow(1)$ as (3) $\Rightarrow(2)$ is shown in Corollary 3.6. By Theorem 4.5 one has $X \tau\left(\mathbb{R} P^{1} \rightarrow \mathbb{R} P^{2}\right)$ which combined with Theorem 4.4 yields $X \tau \mathbb{R} P^{2}$. 
Theorem 4.7 Suppose $X$ is a metrizable space. If $X$ is of finite dimension, then the following conditions are equivalent:
(1) $X \tau \mathbb{R} P^{2}$
(2) $X \tau \mathbb{R} P^{\infty}$

Proof $(1) \Rightarrow(2) \quad$ This follows from Theorem 1.1.

(2) $\Rightarrow(1)$ Notice $\Sigma\left(\mathbb{R} P^{2}\right)$ is a simply connected Moore space $M\left(\mathbf{Z}_{2}, 2\right)$, so applying Theorem 1.2 for metrizable spaces [9] gives $X \tau \Sigma\left(\mathbb{R} P^{2}\right)$. Use Corollary 4.6.

Since $\operatorname{dim}_{\mathbf{Z}_{2}} X \leq 1$ is equivalent to $X \tau \mathbb{R} P^{\infty}$ (in view of $\mathbb{R} P^{\infty}$ being an Eilenberg-Mac Lane space $\left.K\left(\mathbf{Z}_{2}, 1\right)\right)$ we infer the main result of the paper.

Corollary 4.8 Suppose $X$ is a metrizable space. If $X$ is of finite dimension, then the following conditions are equivalent:

(1) $\mathbb{R} P^{2}$ is an absolute extensor of $X$.

(2) $\operatorname{dim}_{\mathbf{Z}_{2}} X \leq 1$.

\section{Appendix}

In this section we prove results on fibrations and bundles that are necessary for the paper.

Proposition 5.1 Suppose $B$ is a finite connected simplicial complex. If $p: E \rightarrow B$ is a circle bundle with fiber $F$, then any map $f: E \rightarrow K(G, 1)$ which is homotopically trivial on a fiber of $p$ factors up to homotopy through $p$.

Proof Notice $E$ is compact. Indeed, as $p: E \rightarrow B$ is a circle bundle, we can cover $E$ with finitely many sets homeomorphic to the product of a simplex of $B$ and the circle $S^{1}$.

Extend the circle bundle to a disk bundle $p^{\prime}: E^{\prime} \rightarrow B$. Take a map $f: E \rightarrow K(G, 1)$ which is homotopically trivial on a fiber of $p$. We will show that $f$ extends continuously to a map $f^{\prime}: E^{\prime} \rightarrow K(G, 1)$. This proves the corollary because $p^{\prime}$ is a homotopy equivalence and therefore $f^{\prime}$ and hence $f$ factor up to homotopy through $B$.

Take a sufficiently fine triangulation of $B$ such that $p^{\prime}$ is trivial over each simplex of $B$. Clearly $f$ extends continuously over $\left(p^{\prime}\right)^{-1}\left(B^{(0)}\right)$. Assume that we already extended $f$ continuously to a map $f^{\prime}: p^{\prime-1}\left(B^{(k)}\right) \cup E \rightarrow K(G, 1)$. Take a $k+1$ simplex $Q$ of $B$. Then $p^{\prime}$ over $Q$ can be represented as the projection from $Q \times D$ to $Q$ with $D$ being a disk. It is clear now that $f^{\prime}$ extends continuously from $\partial(Q \times D)$ over $Q \times D$ and we are done. 
Proposition 5.2 Suppose $p: E \rightarrow B$ is a bundle with compact fiber $F$ that is trivial over $B \backslash C, C$ being closed in $B$. If $f: E \rightarrow K$ is a map such that $f\left(p^{-1}(c)\right)$ is a point for each $c \in C$, then there are maps

$$
p^{\prime}: E \rightarrow \frac{B \times F}{C \times F} \quad \text { and } \quad g: \frac{B \times F}{C \times F} \rightarrow K
$$

such that

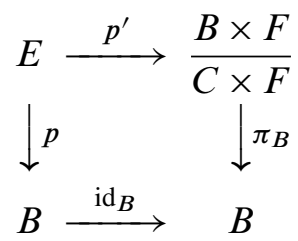

is commutative, $f=g \circ p^{\prime}$, and $p^{\prime}$ is an isomorphism of bundles over $B \backslash C$.

Proof See Definition 3.1 for the definition of $\pi_{B}$. Choose a trivialization

$$
h:(B \backslash C) \times F \rightarrow p^{-1}(B \backslash C)
$$

of $p$ over $B \backslash C$. Define $p^{\prime}: E \rightarrow(B \times F) /(C \times F)$ to equal $q \circ h^{-1}$ on $p^{-1}(B \backslash C)$. If $c \in C$, then put $p^{\prime}\left(p^{-1}(c)\right)=q(\{c\} \times F)$. To prove $p^{\prime}$ is continuous it suffices to show the continuity of $p^{\prime}$ at points in $p^{-1}(C)$. Observe $\pi_{B}$ is a closed map. Indeed, if $A \subset(B \times F) /(C \times F)$, then $\pi_{B}(A)$ equals $\operatorname{proj}_{B}\left(A^{\prime}\right)$, where $A^{\prime}=q^{-1}(A)$. If $A$ is closed, then $A^{\prime}$ is closed, so $\operatorname{proj}_{B}\left(A^{\prime}\right)$ is closed since $\operatorname{proj}_{B}$ is a closed map, being the projection along the compact factor $F$. Therefore any neighborhood of the one-points set $\pi_{B}^{-1}(c), c \in C$, contains $\pi_{B}^{-1}(U)$ for some neighborhood $U$ of $c$ in $B$. Since $\left(p^{\prime}\right)^{-1}\left(\pi_{B}^{-1}(U)\right)=p^{-1}(U)$ is open, then $p^{\prime}$ is continuous at points in $p^{-1}(C)$.

A similar argument shows $p^{\prime}$ is a quotient map $\left(\left(p^{\prime}\right)^{-1}(U)\right.$ is open implies $U$ is open). This time use the tube lemma instead of the fact that $\operatorname{proj}_{B}$ is a closed map. Therefore $f$ factors uniquely as $f=g \circ p^{\prime}$ and $g$ is continuous.

Theorem 5.3 Suppose $B$ is a simplicial complex with the weak topology and $p: E \rightarrow$ $B$ is a map. If $X$ is a metrizable space such that $p^{-1}(\Delta)$ is an absolute extensor of $X$ for all simplices $\Delta$ of $B$, then for any commutative diagram

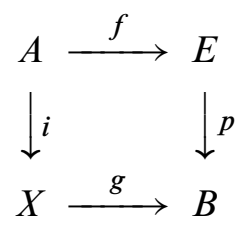

where $A$ is closed in $X$, there is a continuous extension $F: X \rightarrow E$ of $f$ such that $p(F(x))$ and $g(x)$ belong to the same simplex $\Delta_{x}$ of $B$ for all $x \in X$ (in particular, $g$ is homotopic to $p \circ F$ rel. the set of points on which they coincide). 
Proof This is a well-known result in Extension Theory. A special case of it is Proposition 1.2 in [8] that covers the case of $B$ being compact. Theorem 5.3 follows from the special case as each point $x$ of $X$ has a neighborhood $U_{x}$ with $g\left(U_{x}\right)$ contained in a compact subcomplex $K_{x}$ of $B$. Using the special case one can inductively define $F_{\Delta}$ over sets $g^{-1}(\Delta), \Delta$ being a simplex in $B$, and the union of all $F_{\Delta}$ is continuous over each $U_{x}$, hence continuous over $X$.

The following is an application of Theorem 5.3 that is of fundamental importance for this paper:

Corollary 5.4 Suppose $L \neq \varnothing$ is a subcomplex of a connected simplicial complex $K$ and $X$ is a metrizable space such that $X \tau \Sigma(F)$ for some $C W$-complex $F$. Given a closed subset $A$ of $X$ and a map $f: A \rightarrow(K \times F) /(L \times F)$ such that $\pi_{K} \circ f$ extends continuously over $X, f$ extends continuously over $X$.

Proof Recall $\pi_{K}:(K \times F) /(L \times F) \rightarrow K$ is induced by the projection onto the first coordinate. The assumption $L \neq \varnothing$ ensures that the fibers of $\pi_{K}$ are contractible in its domain.

Observation Notice $F$ being contractible in a CW-complex $P$ and $P / F$ being an absolute extensor of $X$ implies $P$ is an absolute extensor of $X$ as the identity map $P \rightarrow P$ factors through $P / F$ up to homotopy. That means we may add a finite number of vertices to $L$ if desired.

Claim 1 If $K$ is a simplex and $L$ is the union of a full subcomplex of $K$ and a subset of vertices $K^{(0)}$ of $K$, then $(K \times F) /(L \times F)$ is an absolute extensor of $X$.

Proof of Claim 1 We will proceed by induction on $\operatorname{dim}(K)$. The first relevant case is $\operatorname{dim}(K)=1$. Notice $(K \times F) /(L \times F)$ is homeomorphic to $K$ if $L=K$, it is homeomorphic to the suspension of $F$ if $L$ consists of one vertex, and is homeomorphic to $\Sigma(F)$ if $L$ consists of two vertices. If $m=\operatorname{dim}(K)>1$ we may assume $L$ contains all the vertices by the Observation above and $L \neq K((K \times F) /(L \times F)$ is homeomorphic to $K$ if $L=K)$. There is a 1 -simplex $e=\left[v_{1}, v_{2}\right]$ of $K$ that does not belong to $L$. Let $v$ be the midpoint of $e$. We can split $K$ into two $m-$ dimensional simplices $\Delta_{1}$ and $\Delta_{2}$ by adding $v$ to the set of vertices of $\Delta$. Let $D_{i}$ be the $(m-1)$-face of $\Delta$ contained in $\Delta_{i}$ for $i=1,2$. Notice the simplicial deformations of $\Delta_{i}$ to $D_{i}$ induce deformation retractions of $\pi_{K}^{-1}\left(\Delta_{i}\right)$ to $\pi_{K}^{-1}\left(D_{i}\right)$. By the inductive assumption we deduce $\pi_{K}^{-1}\left(\Delta_{i}\right), i=1,2$, and $\pi_{K}^{-1}\left(D_{1} \cap D_{2}\right)$ are absolute extensors of $X$. Therefore their union $(K \times F) /(L \times F)$ is an absolute extensor of $X$. That completes the proof of Claim 1. 
If $L$ is the union of a full subcomplex of $K$ and the set of vertices $K^{(0)}$ of $K$, then by Claim 1 the projection $\pi_{K}:(K \times F) /(L \times F) \rightarrow K$ has the property that $\pi_{K}^{-1}(\Delta)$ is an absolute extensor of $X$ for each simplex $\Delta$ of $K$. An application of Theorem 5.3 produces a continuous extension of $f$ in that case. In the general case we may assume $L$ is a full subcomplex of $K$. Since the identity map id on $(K \times F) /(L \times F)$ is null-homotopic on every slice $\{x\} \times F, x \in K$, id factors up to homotopy through $(K \times F) /\left(L_{1} \times F\right)$, where $L_{1}=L \cup K^{(0)}$. By the previous case the composition of $f$ and the natural projection $(K \times F) /(L \times F) \rightarrow(K \times F) /\left(L_{1} \times F\right)$ extends continuously over $X$. That implies $f$ extends continuously over $X$.

Corollary 5.5 Suppose $K$ is a connected simplicial complex and $X$ is a metrizable space such that $X \tau \Sigma(F)$ for some $C W$-complex $F$. Given a closed subset $A$ of $X$ and a map $f: A \rightarrow K \times F$ such that $\pi_{K} \circ f$ extends continuously over $X$, then $u \circ f$ extends continuously over $X$ for any map $u: K \times F \rightarrow M$ to a $C W$-complex $M$ provided $u$ is null-homotopic on $\{v\} \times F$ for some $v \in K$.

Proof Extendability of $u \circ f$ does not depend on the homotopy class of $u$, so we may assume $u$ is constant on $\{w\} \times F$ for some vertex $w$ of $K$. Hence $u$ factors through $(K \times F) /(L \times F), L=\{w\}$, and we may apply Corollary 5.4.

\section{References}

[1] M Cencelj, A N Dranishnikov, Extension of maps to nilpotent spaces. II, Topology Appl. 124 (2002) 77-83 MR1926136

[2] M Cencelj, A N Dranishnikov, Extension of maps into nilpotent spaces. III, Topology Appl. 153 (2005) 208-212 MR2175345

[3] M Cencelj, J Dydak, A Mitra, A Vavpetič, Hurewicz-Serre theorem in extension theory, Fund. Math. 198 (2008) 113-123 MR2369125

[4] M Cencelj, J Dydak, J Smrekar, A Vavpetič, Ž Virk, Compact maps and quasi-finite complexes, Topology Appl. 154 (2007) 3005-3020 MR2355886

[5] A N Dranishnikov, On a problem of P S Aleksandrov, Mat. Sb. (N.S.) 135(177) (1988) 551-557, 560 MR942139

[6] A N Dranishnikov, Extension of mappings into CW-complexes, Mat. Sb. 182 (1991) 1300-1310 MR1133570

[7] A N Dranishnikov, Cohomological dimension theory of compact metric spaces, Preprint, Topology Atlas (1999) Available at http://at.yorku.ca/t/a/i/c/ 43. htm 
[8] A N Dranishnikov, J Dydak, Extension dimension and extension types, Proc. Steklov Inst. Math. 212 (1996) 55-88 MR1635023

[9] J Dydak, Cohomological dimension and metrizable spaces, Trans. Amer. Math. Soc. 337 (1993) 219-234 MR1153013

[10] J Dydak, M Levin, Extensions of maps to the projective plane, Algebr. Geom. Topol. 5 (2005) 1711-1718 MR2186116

[11] M Levin, Some examples in cohomological dimension theory, Pacific J. Math. 202 (2002) 371-378 MR1887771

Department of Mathematics, University of Tennessee

Knoxville, TN 37996-1300, United States

Department of Mathematics, Ben Gurion University of the Negev

P.O.B. 653, Be'er Sheva 84105, Israel

dydak@math.utk.edu, mlevine@math.bgu.ac.il

www. math.utk. edu/ dydak

Received: 4 April 2007 Revised: 22 February 2009 